\title{
The Relationship Between Inhibitory Control and Working Memory and Its Underlying Neural Mechanism
}

\author{
Yibo Peng ${ }^{1, a, *, \dagger}$, Yuhan $\mathrm{Lu}^{2, \mathrm{~b},{ }^{*}, \dagger}$ and Jingwen $\operatorname{Qin}^{3, \mathrm{c}, *, \dagger}$
}

\author{
${ }^{I}$ School of Medicine \& Holistic Integrative Medicine, Nanjing University of Chinese Medicine, Nanjing, Jiangsu \\ Province 210023, China; \\ ${ }^{2}$ School of Psychology, Shaanxi Normal University, Xian, Shaanxi Province 710000, China; \\ ${ }^{3}$ School of Foreign Languages, Shanxi Normal University, Linfen, Shanxi Province 041004, China. \\ *Corresponding author.Email: ${ }^{*} 019018239 @$ njucm.edu.cn, ${ }^{b} l u y u h a n 20011015 @ s n n u . e d u . c n$, \\ c1914020120@stu.sxnu.edu.cn \\ These authors contributed equally
}

\begin{abstract}
Both working memory (WM) and inhibitory control (IC) are the cognitive abilities required for information processing, which have been explored frequently by predecessors. In the classic multicomponent working memory model, the phonological loop, visuospatial sketchpad, and the central executive are three components of working memory. Inhibitory control, also an important ability for problem solving, refers to an attentional control and a response control. As WM is a fundamental resource playing an important role in attributing to other cognition capacities, it is significant to study its mechanism. Such mechanisms have been widely examined by numbers of WM tasks and IC measurements in behaviorism and neuroimaging. Although a number of mechanisms have been proposed in psychology and neurology, there is still no consensus theory to explain the nature of WM and the connection between WM and IC. This review summarized relative to research on the development of the individual, finding a certain association between working memory, inhibitory control, and problem solving. As for the individual with a mental disorder, we suggested that deficient inhibitory control capacity may deteriorate working memory performance. Regarding the neural mechanism, we reviewed studies conducted by fMRI or EEG, finding that working memory and inhibitory control are two independent capacities with some correlations from the view of brain areas. With respect to how IC affects WM, we reviewed research based on EEG and eye dilation and found that updating, a core executive WM functions, is often associated with IC. For future research, as we suggested, causation studies, rather than correlation ones, are significant to better understand the relationship between working memory and inhibitory control. Moreover, more attention is supposed to be paid to the development and trace studies instead of only concentrating on cross-sectional comparisons By and large, to better understand human's cognition activities, it is significant to encode the mechanism of WM and investigate the relationship between WM and IC.
\end{abstract}

Keywords: Working memory, Inhibitory control, Neural mechanism, Cognitive science

\section{INTRODUCTION}

Working memory (WM) is a cognitive ability available to store task-related contents and operate attention-related supervisory when manipulating tasks that require both information processing and memorizing. Reading, arithmetic problem solving are considered as functions of WM because such tasks require temporary storing task-related contents when manipulating. Therefore, individuals with poor WM resources are considered to be less sufficient in solving the problem. Inhibitory control (IC) refers to an attentional control that can access, deleting, and restraining information and includes response control [1]. Individuals with poor IC are assumed to behave less efficiently in problem solving because of insufficiency in distracting irrelevant information and accurately accessing past record memory. For example, individuals with a deficiency in IC generally achieve less academic attainment because that requires capacities such as retrieving information and 
attending to cues from reading or listening materials, which just about their deficiencies. The study also implied that deficiency in IC contributes to intellectually disabled social and interpersonal relationship disorder. When manipulating mathematical problems such as 5 multiplied by 12 , a possible compute mode can retain precalculated digits 50 (5 times 12 's tens place) and 10 (5 times 12's ones place) adding two digits together finally figures up 60. By this instance, we can definitely find a certain correlation between WM and IC when solving problems since these two functional resources are often mobilized at the same time. Interventions aiming at enhancing WM capacity have been researched in various fields [2]. Studying developmental relationships and the nature of WM and IC is crucially important to recognize cognitive patterns of the individual with insufficient WM capacity. Besides, it is also momentous to explore intervention aiming at improving the efficiency of deploying immutable WM capacity in teaching practices and educational and special education to enhance information encoding and retrieving in academic and daily life.

Since the concept of working memory was introduced, it has attracted the attention of researchers in the 1960s [3-5]. Chai and colleagues summarized several working memory studies from the perspective of psychology and neuroscience, refined scientific knowledge about working memory, and gave the latest introduction to the field of working memory [3]. The most famous working memory model is the multicomponent working memory model, which is widely cited in the literature [3]. According to the multicomponent working memory model, there are three components of working memory, namely phonological loop, visuospatial sketchpad, and the central executive, which involves the attentional control system [3-5]. Later, Baddeley added one more component: the name is "episodic buffer" to the working memory model [3]. Besides the multicomponent working memory model, the embedded-process model proposed by Cowan is also a widely cited one, which explored the relationship between short-term memory, long-term memory, and working memory [6,7]. From the perspective of neuroscience, some studies investigated the working memory model at the neuronal level, which explored the associations or dissociations with the different systems of working memory $[3,8]$. It is believed that working memory lays the foundation for many other cognitive controls in humans and decoding the working memory mechanism will be the first step to promote the understanding of other aspects of human cognition. Subsequently, the interaction between working memory and other cognitive systems can be reasonably examined [5]. As for the working memory tasks, according to Chai and colleagues, due to the limitation of the nature of work, working memory research is difficult to reach a consensus on research programs [3]. Hence, there are plenty of working memory tasks (e.g., Sternberg task, nback task, Corsi block-tapping test, Wechsler's Memory Scale (WMS), and working memory subtests in the Wechsler Adult Intelligence Scale (WAIS) - Digit Span, Letter Number Sequencing) [3]. Inhibition control, in which the frontal cortex plays an important role, has substantial experiment paradigms (e.g., go-no-go, stopsignal, inhibition of return, negative priming, Stroop, and card sorting paradigm) [9]. As for the methods to assess the efficiency of inhibition control, reaction time (RT) and error rate are typical. Regarding the neuroimaging studies on inhibition control, results turn out that inhibitory control is subserved by the frontal cortex $[10,11]$. Considering that the structure and function of the frontal cortex will change throughout the life cycle, one can predict the accompanying changes in inhibitory control. Many researchers have explored the relationship between inhibitory control and the life span. Both the Stroop color-word task conducted by Comalli and colleagues and the stop-signal task conducted by Williams et al. demonstrated that inhibitory control improves during childhood and declines during late adulthood [10,12]. Regarding the changes in IC during the life span, a color-word Stroop experiment performed on the participants ranging from childhood to late adulthood has been conducted. It was found that the response speed under incongruent condition, which is negatively correlated with one's inhibition control, decreased in children aged 7 to 19 years, stabilized in middle-aged adults, and increased in elderly people aged 65 to 80 years [12]. Williams et al. and colleagues also concluded that inhibition control improves in childhood but decreases in later adulthood [13].

WM, which has already proved to alter gradually over the development in lifespan, is closely related to information processing and attention resources. And a large body of hypothesis about mechanisms and tasks behind the working memory have been proposed and studied thoroughly to some extent but not have a consensus theory till now. It has been already researched that the frontal cortex is thought to play a particularly important role in both working memory and executive abilities, and IC has a close relationship with attention resource filtering and concentration. It has been proved that IC also can be gradually altered during life span [11]. However, the aspects including the inner connections between WM and IC have not been extensively studied in the area. Still, it is obvious that both processes are associated with psychological and neuroscience functions in attention distribution, information processing, and memory focus and have always been connected as the reason behind mental disorder diseases. This article seeks to demonstrate and outline several up-to-date overview studies that are related to this gap and discuss them in respect of WM and IC from the following aspects: development, abnormal population contrast, and neuroscience perspective in an effort to refine and unite 
the scientific knowledge about the connection between both sides and point out some areas further researches required.

\section{A DEVELOPMENTAL PERSPECTIVE OF THE RELATIONSHIP BETWEEN WM AND IC}

Problem solving refers to cognitive processing that is directed to a certain goal by processing procedures, including representing and manipulating based on the problem solver's knowledge and skill reserve [14]. According to a previous study, deficient WM and IC were attributed to poor problem-solving performance in children. Numerical or linguistic WM tasks were conducted between good problem solvers and poor numerical problem solvers across fourth-grade children to investigate the mechanism behind impaired WM [15]. The result shows that children with poor problem-solving skills make more intrusion errors in numerical and linguistic WM tasks than good problem solvers, indicating poor problem solvers insufficient IC to ignore irrelevantly or information. Besides, the results also mirror that children with poor problem-solving skills lack flexibility in manipulating both verbal and numerical items because of poor WM capacity, according to Baddeley's central executive component in the WM model [5]. Although the relationship between WM and IC in childhood is not clear according to their study, they indicated poor problem-solving performance because of immature cognitive function in childhood do relate to insufficient IC and poor WM capacity. To further understand the relationship between WM and IC, a large number of studies explore it in the view of life span.

Childhood has been interpreted as a developmental period that full of plasticity, manifesting insufficient WM performance and deficiencies in IC, memory capacity, and emotional control compared to the adult. Before entering childhood, preliminary development of WM capacity was found, suggesting a continuous cognitive growth from infant to a child [16]. The developmental change and relationship between WM and IC among children and early adolescents aged 6-17 were investigated by following tasks [17]. The memory span paradigm for measuring WM requires participants to remember items and their order, Stroop task paradigm demands that participants react in accord or in opposition according to certain cues to measure WM+IC. Finding that efficiency score is less in younger children than elder children in two measurements, indicating that there is a steeper developmental improvement both in WM and $\mathrm{WM}+\mathrm{IC}$, rather than the capacity that just storing items, which confirmed chronological age alone is significantly related to the efficiency of WM. The nature of developmental changes of WM and IC during childhood was explained in terms of cognitive science, as children lack strategic knowledge to stay focused and the ability to suppress irrelevant items, owing to the undeveloped prefrontal cortex. Neurologically, they implied left prefrontal cortex attributed to WM and IC efficiency, which was considered mature in the elder period [18]. As for the relationship between WM and IC, since an adequate source of WM capacity was found able to retain task-relevant items while suppressing irrelevant items in the process of growth, they suggested that the association between WM and IC tends to become stronger during childhood.

As cognition level and the association between WM and IC show a boost during childhood and young adulthood, we ponder on when will cognitive level and association decline in a later period. In the study by Christelle et al., series of Reading span tests aiming to investigate the relationship between WM and IC were conducted among different age groups, from children whose average age is 11.36 to an elder adult whose average age is 75.38 [19]. Reading span test, a typical experiment to measure $\mathrm{WM}$, requires participants to desire whether the sentence is true or false and retain the last word of each trial. In this study, two versions of the Reading span test were conducted, and participants were classified into children, young adults, old-young adults, and old-old adults. One version required participants to decide whether the semantic of the sentence is correct then recall the final word of each trial in sequence, and another required participants to do a version of the Reading span experiment that one trial more than their WM level. Besides, they analyze different kinds of intrusion errors, respectively, the previous trail word, current trail word instead of the target word, and word that is not presented in the experiment. As a result, it is found that children make more mistakes than younger adults, which is consistent with biological neurology developmental of the prefrontal cortex has not been fully developed [18]. As for older adults, it is found that they produce more previous-list intrusion because they have greater difficulty in distinguishing relevant and irrelevant items. Moreover, researchers also implied IC function for suppressing irrelevant information and WM performance increases in tandem in childhood and declines in elder adulthood but has not stated the correlation in between.

In summary, it was found that WM and IC's deficiency do affect problem solving, and by versions of separate or combined WM and IC experiments, a different correlation between WM and IC was found across the life span. Such developmental change has been interpreted as an attentional resource that accounts the same task could be skill-drain for child and older adult. Another interpretation is that inhibit capacity increase during childhood then become lessen in elderly adult [20,21]. Although various models and hypotheses were presented, WM and IC respectively change over the course of a lifetime. Very few studies probe the inner correlation between WM and IC in the view of life span. 


\section{ABNORMAL DEVELOPMENT OF WM OR IC AND ITS IMPACT}

Reading disability has been conceptualized as consisting of appearing defect in pronunciation awareness, nominate ability, pronunciation memory, and character processing [22]. Reading skills includes a system that remembers former specific information about the words and processes them in order to shape a whole comprehension later, and WM, which involves temporary storage of information and retrieval information from long-term memory, is thought to have a relationship with a number of cognitive processes as a central executive, such as reading, problem solving and learning skills $[23,24]$. The relationships among WM, IC, and reading disability were studied in an experiment by providing a listening span task. The experimenter read some sentences to individuals, and each of them was missing its final word, individuals guess the missing word and attempted to repeat all the final missing words on completion of the set, and none of the individuals experienced difficulties in providing the losing word [25]. No response and the intrusion errors (miscalling a target word) were analyzed, and intrusion errors were divided into three types: recalling words from the current trial but not the target, recalling early trail words, and recalling words that have not been presented, each type of errors were used as dependent variables. The result shows that deficits in IC's access and restraint functions may contribute to the difficulties experienced by disabled readers in WM [25]. IC may prevent goal-irrelevant information from entering WM and participate in deleting and removing information that exists but is no longer important in WM. Scientists also found that WM will decline along with aging, and it may result from deficits in the inability to filter from WM information that is irrelevant or information that is no longer relevant, and this is affected by IC.

OCD is associated with highly unpleasant obsessive thoughts and compulsive behaviors in most patients and is related to cognitive dysfunctions, mainly in executive functions and complex memory tasks such as WM updating [26,27]. A recent hypothesis explaining OCDrelated alterations in brain activations and performance in WM has suggested that impaired WM in OCD may associate with difficulties in concentrating on the relevant items and failures to inhibit and prevent irrelevant stimuli [28]. SMA, IPL, and IFG have been reported to show aberrant activations in OCD during WM as well as inhibitory control tasks [29]. So, the WM declining may also affect the IC ability development in OCD patients. An experiment asked OCD and health participants to perform numeric N-BACK task (watching the line of numbers and reacting if the number is the same as front ones, pressing the button if the number is the same as the last one in 1-back task, 2-back, 3-back are in the similar procedure) with four WM load conditions during functional Magnetic Resonance Imaging (fMRI). And individuals are also needed to finish the stop-signal task (SSRT) to ignore the auditory signal and do the motor response [30]. The result shows that faster responses in $\mathrm{n}$-back are related to lower SSRTs (presenting a higher inhibition performance). Patients with OCD showed WM performance decrements, specifically at high WM load that were accompanied by WM load-dependent alterations in neural activity in SMA and IPL as well as reduced response IC [30]. There was a significant relationship between SSRT and SMA-activity during 0-back,1-back ,2-back, and 3-back, which shows that higher SMA-activity is related to lower SSRT values and better inhibition performance in OCD. It means that a high level of WM ability and related brain activities have a relationship with higher inhibition performance in OCD.

Patients with Alzheimer's disease have an impairment of inhibitory control for reasons that are currently still unclear. Patients with early Alzheimer's disease usually present with a symptom of a deterioration in episodic memory, executive function, and attention, including the IC of cognition and behaviour [31]. An experiment is showed to explore whether WM and IC is a bidirectional influence or not and eye-tracking has been used as a tool and Alzheimer and healthy aging individuals need to complete the Anti-saccade task gap and overlap (they need to separately look away and look forward to the direction opposite the target after starting with central fixation and then turn back to the central square surround) [31]. MMSE tested participants` verbal working memory and spatial working memory before the main experiment. Experimenters recorded eye movements and analyzed some types of errors, such as the number of inhibition errors and uncorrected errors in different groups. The result shows that on the MMSE, the AD showed greater impairment on the cognitive scores than $\mathrm{OC}$, and there was a significant increase in $\mathrm{AD}$ inhibition errors and target omissions compared to health aging groups. There was no fundamental sensorimotor deficit in spatial programming, processing speed, and motivation between the $\mathrm{OC}$ and $\mathrm{AD}$. Still, there was a significant correlation between the frequency of uncorrected errors and both the spatial working memory and verbal working memory in AD. Studies have argued that working memory is critically important for inhibitory control in the AST. Using the dual-task methodology, several studies have shown that tasks imposing a load on working memory will disrupt eye movements in the AST [32]. Studies suggested that people with AD have particular difficulty in the AST because the attention for tracking the target competes with the required attention to monitor and correctly recall the intended location of their eye movements at the same time. Thus, the deterioration in spatial working memory is an important contributory factor in the account of uncorrected errors about IC in 
AD. The IC and WM are bidirectionally affecting each other.

\section{NEURAL MECHANISM UNDERLYING THE RELATIONSHIP}

The relationship between WM and IC is a hot topic of research that many researchers are curious whether the two are related or independent. Many behavioral studies suggested that WM and IC are interrelated functions $[30,31]$. For example, the famous conservation-ofnumber task, an example of the inability of children to separate the number of objects in arrays from their spatial extents, designed by Piaget, suggested that both the ability to acquire knowledge and the one to inhibit previous knowledge are important for problem solving [33]. Neo-Piagetians also found that cognitive development is related to WM and IC [33,34]. For example, an fMRI study found that the number conservation principle may be directly related to the efficiency of IC, which allows people to inhibit false intuitive perception heuristic responses [30,35]. Moreover, another fMRI study demonstrated that activations in the right insula/inferior frontal gyrus, a region critical to IC, were elicited in the number conservation task. In other words, the results of these studies turned out that IC is related to WM when an individual is conducting cognitive tasks [30]. It is consistent with the neuroscience research that both IC and manipulating information in WM relies on the inferior portions of the frontal lobes [30,36].

As mentioned above, the correlation between WM and IC was different across the life span; for the abnormal individual, IC and WM are bidirectionally affecting each other. The results from predecessors seem to be controversial, and the exact relationship between them is still unclear. Mecklinger and colleagues researched the relationship between inhibitory control and working memory capacity by fMRI and behavioral methods, finding that though participants with high WM capacity were better at deal with interference, the brain regions responsible for IC and WM sometimes are not consistent $[32,38]$. As for verbal material recognition, left PFC regions, which is related to both WM and IC, are activated; however, for other forms of recognition tasks, right PFC regions also engage in IC but WM [32]. IC is a general non-mnemonic control function that is mediated by a specific PFC region, which is different from WM [32]. In the study of Mecklinger and colleagues, instead of determined, WM modulated the interference effects in the letter task [38]. Hence, no matter be based on the results of behavioral studies or the activation of brain regions, it is safe to conclude that IC and WM are two independent capacities with some associations.

Although the relationship between IC and WM has been found, it is still unclear as to the specific aspects of IC affecting WM. As mentioned above, attention control is the mutual influence between WM and IC. Besides, from the perspective of executive functions, WM is also influenced by IC. There are 3 core executive WM functions (EFs): updating, shifting, and inhibition [40]. According to this definition, one of the executive WM functions, inhibition, is overlap with IC. Updating, referring to processes of retrieval, transformation, and substitution of WM content, are often controlled by the $\mathrm{n}$ back task. It is not clear whether IC has any association with updating. In the study of Scharinger and colleagues, by applying the n-back task paradigm, the interaction between inhibitory demands and load on WM are explored, conducted by behavioral measures, EEG, and pupil dilation. It was found that increased load on updating of WM resulted in the enhancement of IC [39]. In other words, the activation of updating is often accompanied by improved IC. Miyake et al. reached a similar conclusion that updating, a core executive WM function, shares underlying network structure serving controlled attention with IC [40]. Moreover, in the EEG study conducted by Scharinger and colleagues, a decrease of the P300 amplitude was found when different WM functions were necessary for task performance. This may be because attention was distributed to IC and updating [39]. As a result, this is manifested by the high correlation between WM and IC. In summary, updating is the part of WM functions to be affected by IC, and this further reveals that IC and WM are two independent but related mechanisms.

\section{LIMITATION AND FUTURE DIRECTION}

Not surprisingly, with so many aspects of working memory and inhibitory control correlations yet to be understood and its growing complexity, the exact causal link between WM and IC basis has not been studied enough. Current studies were more concentrated on cross-sectional comparisons, but development and trace studies were not enough. In practice, even though all of the aforementioned studies contribute to the effect of separately changing one aspect and these were widely established by employing various methodologies to study it, it has been postulated that there were not many studies about interfering with one of them and seeing how the other changed. Further research is required to explore the exact causal link between WM and IC basis before an exhaustive account can be gathered. Tracing and development studies can be used to identify how the relationship between WM and IC change among different stages in different groups such as OCD and Parkinson in latency period and the stage when patients` symptoms were appearing. The need to interfere with one of them and explore whether the other change and the degree of change can also be dissected in further research (whether the increase of WM ability can lead to less decline in IC). 


\section{CONCLUSION}

By investigating the WM capacity of children, the study has found that problem solving performance is associated with insufficient IC and poor WM capacity. Insufficient WM performance in childhood was interpreted as lacking strategic knowledge because of the undeveloped prefrontal cortex, and the brain region matures as an individual grows. Nevertheless, as the individual grows older, the cognitive level will be seen a peak in young adulthood and make them more susceptible to proactive memory, owing to decline in cognition function of IC. Since deficient IC capacity can lead to disability in tasks, psychologists and neurologists also require cognitive resources, psychologists, and neurologists through light on abnormal development of WM and IC. Studies have found that deficits in the access and restraint functions of IC can contribute to reading disability. Insufficient WM performance was found in patients with OCD because as activity of SMA and IPS changed along with WM load results in decrements of IC. Besides, it was found that spatial WM significantly accounts for greater impairment of IC in AD and can further decipher the bilingual influences between WM and IC. A fMRI study indicated that WM and IC are two interrelated functions relying on the inferior portions of the prefrontal lobe. Yet IC was found mediated by specific brain regions different from WM confirmed by fMRI and behavioral methods, indicating that although these cognitive resources correlate, it is also concluded that they are independent capacities. Updating as a function of WM was also found to share the same underlying network as IC, suggesting that WM and IC are related and independent. To sum up, since WM plays an important role in cognition activities, encoding the mechanism behind it is significant to understand nature. It is expected to explore further in the future.

\section{REFERENCES}

[1] Hasher, L., Zacks, R. T., \& May, C. P. (1999). 23 Inhibitory Control, Circadian Arousal, and Age'.

[2] Dehn, M. J. (2011). Working memory and academic learning: Assessment and intervention. John Wiley $\&$ Sons.

[3] Chai, W. J., Abd Hamid, A. I., \& Abdullah, J. M. (2018). Working memory from the psychological and neurosciences perspectives: a review. Frontiers in psychology, 9, 401.

[4] D'Esposito, M., \& Postle, B. R. (2015). The cognitive neuroscience of working memory. Annual review of psychology, 66, 115-142.

[5] Baddeley, A. (2010). Working memory. Current biology, 20(4), R136-R140.
[6] Cowan, N. (1999). "An embedded-processes model of working memory," in Models of Working Memory: Mechanisms of Active Maintenance and Executive Control, eds A. Miyake and P. Shah (Cambridge: Cambridge University Press).

[7] Cowan, N. (2005). Working memory capacity. Exp. Psychol. 54, 245-246.

[8] Baddeley, A. D. (2000). Short-Term and Working Memory. The Oxford Handbook of Memory. Oxford: Oxford University Press.

[9] Christ, S. E., White, D. A., Mandernach, T., \& Keys, B. A. (2001). Inhibitory control across the life span. Developmental neuropsychology, 20(3), 653-669.

[10] Casey, B. J., Trainor, R. J., Orendi, J. L., Schubert, A. B., Nystrom, L. E., Giedd, J. N., ... \& Rapoport, J. L. (1997). A developmental functional MRI study of prefrontal activation during performance of a gono-go task. Journal of cognitive neuroscience, 9(6), 835-847.

[11] Konishi, S., Kawazu, M., Uchida, I., Kikyo, H., Asakura, I., \& Miyashita, Y. (1999). Contribution of working memory to transient activation in human inferior prefrontal cortex during performance of the Wisconsin Card Sorting Test. Cerebral Cortex, 9(7), 745-753.

[12] Comalli Jr, P. E., Wapner, S., \& Werner, H. (1962). Interference effects of Stroop color-word test in childhood, adulthood, and aging. The Journal of genetic psychology, 100(1), 47-53.

[13] Williams, B. R., Ponesse, J. S., Schachar, R. J., Logan, G. D., \& Tannock, R. (1999). Development of inhibitory control across the life span. Developmental psychology, 35(1), 205.

[14] Mayer, R. E., \& Wittrock, M. C. (2006). Problem solving. Handbook of educational psychology, 2, 287-303.

[15] Passolunghi, M. C., \& Siegel, L. S. (2001). Shortterm memory, working memory, and inhibitory control in children with difficulties in arithmetic problem solving. Journal of experimental child psychology, 80(1), 44-57.

[16] Reznick, J. S., Morrow, J. D., Goldman, B. D., \& Snyder, J. (2004). The onset of working memory in infants. Infancy, 6(1), 145-154.

[17] Roncadin, C., Pascual-Leone, J., Rich, J. B., \& Dennis, M. (2007). Developmental relations between working memory and inhibitory control. Journal of the International Neuropsychological Society: JINS, 13(1), 59. 
[18] Gogtay, N., Giedd, J. N., Lusk, L., Hayashi, K. M., Greenstein, D., Vaituzis, A. C., ... \& Thompson, P. M. (2004). Dynamic mapping of human cortical development during childhood through early adulthood. Proceedings of the National Academy of Sciences, 101(21), 8174-8179.

[19] Robert, C., Borella, E., Fagot, D., Lecerf, T., \& De Ribaupierre, A. (2009). Working memory and inhibitory control across the life span: Intrusion errors in the Reading Span Test. Memory \& cognition, 37(3), 336-345.

[20] De Ribaupierre, A. (2001). Working memory and attentional processes across the lifespan. Lifespan development of human memory, 59-80.

[21] Dempster, F. N. (1981). Memory span: Sources of individual and developmental differences. Psychological Bulletin, 89(1), 63.

[22] Liu, F. F., Zuo, P. X., Tang S. T., Gao, X. Y. , He, H. Y. Research progress on neural mechanism of orthographic processing in children with Chinese developmental dyslexia. Chinese Journal of School Health(03),465-470.

[23] Baddeley, A. D. (1983). Working memory. Philosophical Transactions of the Royal Society of London: Series B, 302, 311-324.

[24] Baddeley, A. D (1986). Working memory. London: Oxford University Press.

[25] Chiappe, P., Hasher, L., \& Siegel, L. S. (2000). Working memory, inhibitory control, and reading disability. Memory \& cognition, 28(1), 8-17.

[26] Harkin, B., \& Kessler, K. (2011). The role of working memory in compulsive checking and OCD: a systematic classification of 58 experimental findings. Clinical psychology review, 31(6), 10041021.

[27] Mendlowicz, M.V., Stein, M.B. (2000). Quality of life in individuals with anxiety disorders. The American Journal of Psychiatry ,157, 669-682.

[28] de Vries, F. E., de Wit, S. J., Cath, D. C., van der Werf, Y. D., van der Borden, V., van Rossum, T. B., van Balkom, A. J., van der Wee, N. J., Veltman, D. J., \& van den Heuvel, O. A. (2014). Compensatory frontoparietal activity during working memory: an endophenotype of obsessive-compulsive disorder. Biological psychiatry, 76(11), 878-887.

[29] Nee, D.E., Brown, J.W., Askren, M.K., Berman, M.G., Demiralp, E., Krawitz, A., Jonides,J. (2013) - A meta-analysis of executive components of working memory. Cereb. Cortex N. Y. N 1991 (23), 264-282.
[30] Heinzel, S., Kaufmann, C., Grützmann, R., Hummel, R., Klawohn, J., Riesel, A., Bey, K., Lennertz, L., Wagner, M., \& Kathmann, N. (2017). Neural correlates of working memory deficits and associations to response inhibition in obsessive compulsive disorder. NeuroImage. Clinical, 17, 426-434.

[31] Greenwood PM, Parasuraman R, Alexander GE (1997). Controlling the focus of spatial attention during visual search:effects of advanced aging and Alzheimer's disease. Neuropsychology 11:3-12

[32] Crawford, T.J., Higham, S., Mayes, J. et al. The role of working memory and attentional disengagement on inhibitory control: effects of aging and Alzheimer's disease. AGE 35, 1637-1650 (2013).

[33] Greenwood PM, Parasuraman R, Alexander GE (1997) Con-trolling the focus of spatial attention during visual search:effects of advanced aging and Alzheimer's disease. Neuropsychology 11:3-12

[34] Ping, R. M., \& Goldin-Meadow, S. (2008). Hands in the air: Using ungrounded iconic gestures to teach children conservation of quantity. Developmental psychology, 44(5), 1277.

[35] Deary, I. J., Penke, L., \& Johnson, W. (2010). The neuroscience of human intelligence differences. Nature reviews neuroscience, 11(3), 201-211.

[36] Wright, I., Waterman, M., Prescott, H., \& Murdoch - Eaton, D. (2003). A new Stroop - like measure of inhibitory function development: Typical developmental trends. Journal of Child Psychology and Psychiatry, 44(4), 561-575.

[37] Mecklinger, A., Weber, K., Gunter, T. C., \& Engle, R. W. (2003). Dissociable brain mechanisms for inhibitory control: effects of interference content and working memory capacity. Cognitive Brain Research, 18(1), 26-38.

[38] Poirel, N., Borst, G., Simon, G., Rossi, S., Cassotti, M., Pineau, A., \& Houdé, O. (2012). Number conservation is related to children's prefrontal inhibitory control: an fMRI study of a Piagetian task. PloS one, 7(7), e40802.

[39] Davidson, M. C., Amso, D., Anderson, L. C., \& Diamond, A. (2006). Development of cognitive control and executive functions from 4 to 13 years: Evidence from manipulations of memory, inhibition, and task switching. Neuropsychologia, 44(11), 2037-2078.

[40] Diamond, A., Barnett, W. S., Thomas, J., \& Munro, S. (2007). Preschool program improves cognitive control. Science (New York, NY), 318(5855), 1387. 